\title{
NUMERICAL STUDIES OF HOMOGENIZATION UNDER A FAST CELLULAR FLOW.
}

\author{
GAUTAM IYER AND KONSTANTINOS C. ZYGALAKIS
}

\begin{abstract}
We consider a two dimensional particle diffusing in the presence of a fast cellular flow confined to a finite domain. If the flow amplitude $A$ is held fixed, and the number of cells $L^{2} \rightarrow \infty$, then problem homogenizes, and has been well studied. Also well studied is the limit when $L$ is fixed, and $A \rightarrow \infty$. In this case the solution averages along stream lines. The double limit as both the flow amplitude $A \rightarrow \infty$ and the number of cells $L^{2} \rightarrow \infty$ was recently studied [9], one observes a sharp transition between the homogenization and averaging regimes occurring at $A \approx L^{4}$. This paper numerically studies a few theoretically unresolved aspects of this problem when both $A$ and $L$ are large that were left open in [9] using the numerical method devised in [16]. Our treatment of the numerical method uses recent developments in the theory of modified equations for numerical integrators of SDEs [23].
\end{abstract}

\section{INTRODUCTION}

A passive tracer diffusing in the presence of a fast cellular flow obeys an SDE of the form

$$
d X=A v(X) d t+d W_{t} .
$$

Here $A>0$ is the magnitude of the velocity (more precisely, $A$ is the Péclet number), and $W$ is a standard (2D) Brownian motion. For simplicity and concreteness, we assume

$$
v(x)=\nabla^{\perp} H \stackrel{\text { def }}{=}\left(\begin{array}{c}
-\partial_{2} H \\
\partial_{1} H
\end{array}\right), \quad \text { where } H\left(x_{1}, x_{2}\right) \stackrel{\text { def }}{=} \frac{1}{2 \pi} \sin \left(2 \pi x_{1}\right) \sin \left(2 \pi x_{2}\right) .
$$

Classical homogenization results $[1,10,15]$ guarantee that on large time scales the particle behaves like a Brownian motion, rescaled by an effective diffusion matrix. That is, for $t$ large, the law of $X_{t}$ is comparable to the law of $\sqrt{D_{\text {eff }}} W_{t}$, where $D_{\text {eff }}$, the effective diffusivity, is a constant matrix. The effective diffusivity can be obtained by solving a cell problem [4,5], and it's asymptotic behaviour as $A \rightarrow \infty$ is like $\sqrt{A} c_{0} I$, where $I$ is the identity matrix and $c_{0}>0$ is a constant $($ see $[5,13,22])$.

On shorter time scales the flow term is dominant, and a different behaviour is observed. As $A \rightarrow \infty$, drift rapidly transports the passive tracer along level sets of $H$, and noise slowly diffuses the tracer across level sets. This results in behaviour that is averaged on level sets (more precisely, on the Reeb graph of the Hamiltonian), has been extensively studied by $[7,12]$ using the theory of large deviations, and by $[2,19]$ using PDE methods.

GI was partially supported by NSF-DMS 1007914 and the Center for Nonlinear Analysis.

KZ thanks the Center for Nonlinear Analysis and Carnegie Mellon University for their hospitality. KZ was supported by Award No. KUK-C1-013-04 of the King Abdullah University of Science and Technology (KAUST). 
Recently the transition between the averaged and homogenized behaviour was studied in [9], by confining the rapidly advected passive tracer confined to a large domain $\Omega$. The trajectories of $X$ (confined to $\Omega$ ) exhibit qualitatively different behaviour dependent on the relative size of the Péclet number, $A$, to the domain size, $L$. Theorem 1.3 in [9] shows that when $A \gg L^{4}$, trajectories of $X$ exit the domain immediately after reaches a cell boundary, forcing 'ballistic' travel along separatrices. On the other hand, when $A \ll L^{4}$, the expected exit time of $X$ from $\Omega$ is comparable to that of the effective Brownian motion, suggesting that $X$ exhibits a homogenized behaviour.

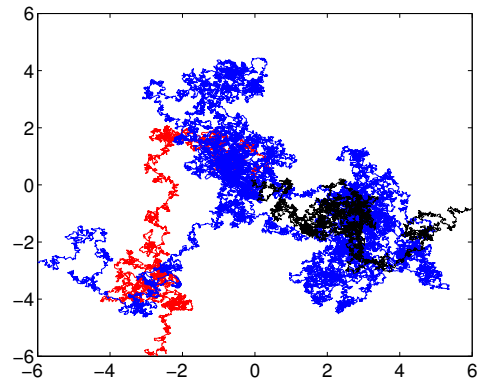

(a) Small amplitude

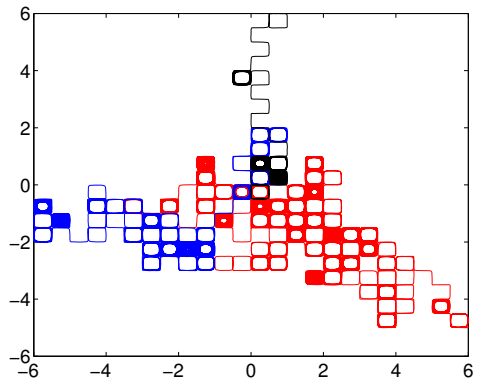

(b) Large amplitude

FigurE 1. Trajectories of three realizations of the diffusion (1.1).

The different qualitative behaviour observed in [9] is nicely illustrated in Figure 1. For a small Péclet number relative to the number of cells (precisely $A=L=10$ ), Figure 1(a) shows trajectories of $X$ resembling that of an effective Brownian motion. For a large Péclet number relative to the number of cells (precisely $A=L^{4.5}, L=$ 10), Figure 1(b) shows multiple trajectories of $X$ which move 'ballistically' along cell boundaries.

The aim of this paper is to numerically study two open questions that arise in [9]. The first question we study numerically is the critical case $A \approx L^{4}$. The methods in [9] address the cases $A \gg L^{4}$, and $A \ll L^{4}$, however, are unable to address the situation where $A \approx L^{4}$. Our numerical simulations (presented in Section 3) suggest that in the critical case $A \approx L^{4}$ the law of the exit time of $X$ appears identical to the distribution of the effective Brownian motion. Consequently, one is led to believe that $A \approx L^{4}$ might still be in the homogenization regime. This is of course not contradictory to [9], because the precise results proved there involve logarithmic corrections in the relationship between $A$ and $L$ (see Theorem 2.1, below).

Another implication of our numerics is regarding the time scale at which $X$ homogenizes. Studying trajectories of $X$, we see that when $A=L^{4}$, a very small fraction trajectories travel 'ballistically' and exit a domain of size $L$ almost immediately (see [21] for a related anomalous diffusion effect). If we throw away this ballistic trajectories, then the remainder behave exactly like an effective Brownian motion. Specifically, in Section 3 we also compare the time these trajectories take to exit a domain of size $L$, to the exit time of an effective Brownian motion and find a remarkable agreement. It is of course well known that $X$ behaves like an 
effective Brownian motion on large time scales. However, the observations above suggest that for large $A$, the process $X$ homogenizes on time scales of order 1 .

The second question we study numerically is an issue left open in [9] when $A \ll$ $L^{4}$. If we study the expected exit time of $X$ from a disk, then [9, Proposition 1.5] shows that as $A, L \rightarrow \infty$, the expected exit time converges to the expected exit time of an effective Brownian motion from the same disk. However, if we consider the expected exit time of $X$ from any other domain, then the results in [9] show that as $A, L \rightarrow \infty$, the expected exit time is only comparable to the expected exit time of an effective Brownian motion, as $A, L \rightarrow \infty$. The failure to obtain an exact limit is a non-trivial obstruction stemming from extraneous terms in the asymptotic expansion. Our numerical simulations (presented in Section 4) suggest that despite theoretical limitations, the expected exit time of $X$ from a square is exactly the expected exit time of the effective Brownian motion.

Finally, we remark on the numerical method used for our simulations. Equation (1.1) is stiff, and when $A$ is large the standard Euler-Maruyama method requires a time step which is too small to be practically useful. We instead use the method developed in [16]. We conclude the paper, with a description of the numerical method and a new, simpler, analysis of it based on [23], which extends the theory of modified equations [20] to more general numerical integrators than the Euler-Maruyama method.

1.1. Plan of this paper. In Section 2, we state a few results from [9], and provide a brief heuristic explanation. In Section 3 we present our numerical results in the critical regime $A \approx L^{4}$. In Section 4 we describe the issues in the homogenization regime $A \ll L^{4}$, that could not be addressed by the methods in [9], and present our numerical results. Finally, we conclude the paper with a description of the numerical method we used to perform our simulations.

\section{The HomogenizATion AND AVERAGing REgimes}

We devote this section to stating the results from [9] concerning the homogenization and averaging regimes. Let $\tau$ be the exit time of $X$ (defined in (1.1)) from the domain $\Omega$, and let $\bar{\tau}(x) \stackrel{\text { def }}{=} E^{x} \tau$ denote the expectation of $\tau$. It is well known (see for instance [14]) that the expected exit time from the domain $\Omega$ satisfies the Poisson problem

$$
\left\{\begin{aligned}
-\frac{1}{2} \Delta \bar{\tau}+A v \cdot \nabla \bar{\tau} & =1 & & \text { in } \Omega, \\
\bar{\tau} & =0 & & \text { on } \partial \Omega,
\end{aligned}\right.
$$

The results in [9] concern the limit of $\bar{\tau}$ and the principal eigenvalue of the operator $-\Delta+A v \cdot \nabla$, as both the Péclet number and the size of the domain $\Omega$ go to infinity. We result for the expected exit time (stated below) shows that the limit of $\bar{\tau}$ is either that of the homogenized equations, or that of the averaged equations with a sharp transition at $A \approx L^{4}$.

Theorem 2.1 ([9]). Let $\Omega=(-L / 2, L / 2)^{2}$ be a square of side length $L$, and $\bar{\tau}=\bar{\tau}_{A, L}$ be the solution to $(2.1)$. 
(a) Suppose $L \rightarrow \infty$, and $A=A(L)$ varies so that $A \approx L^{4-\alpha}$, for some $\alpha \in(0,4)$. There exists a constant $C=C(\alpha)>0$, independent of $A$ and $L$, such that

$$
\frac{1}{C} \frac{L^{2}}{\sqrt{A}} \bar{\tau}(x) \leqslant C \frac{L^{2}}{\sqrt{A}}, \quad \text { whenever }|x| \leqslant(1-\delta) \frac{L}{2}
$$

Consequently as $L \rightarrow \infty$, we have $\bar{\tau} \rightarrow \infty$ uniformly on compact sets.

(b) On the other hand, suppose $A \rightarrow \infty$, and $L=L(A)$ varies so that $A \gg L^{4}$ (more precisely, we need $\left.\sqrt{A} /\left(L^{2} \log A \log L\right) \rightarrow \infty\right)$. There exists a constant $C>0$, independent of $A$ and $L$, such that

$$
\bar{\tau}(x)^{2} \leqslant C \frac{L^{2}}{\sqrt{A}} \log A \log L, \quad \text { whenever } H(x)=0 .
$$

Consequently, if $H(x)=0$, then $\bar{\tau}(x) \rightarrow 0$ as $A \rightarrow \infty$, and $\|\bar{\tau}\|_{L^{\infty}(D)}$ is bounded uniformly in $A$.

A heuristic explanation for the result in Theorem 2.1 can be obtained as follows. First if $A$ is much smaller than $L$ (for instance, if $A$ is fixed and $L \rightarrow \infty$ ), then we expect homogenization to work. Thus, $X_{t} \approx \sqrt{D_{\text {eff }}} W_{t}$, and so we expect $\bar{\tau} \approx$ $L^{2} / D_{\text {eff }} \approx L^{2} / \sqrt{A}$ away from $\partial \Omega$, which is exactly the content of $(2.2)$.

Next if $A$ is much larger than $L$ (for instance, if $A \rightarrow \infty$ and $L$ is fixed), then we expect $X$ to behave like a diffusion averaged on level sets of $H$. Since both the boundary of the big domain $\Omega$, and the boundary of every cell are on the same level set of $H$, we expect the diffusion $X$ to exit immediately from cell boundaries. This is exactly equation (2.3), when $X$ starts on cell boundaries.

The above argument also provides a intuitive explanation of why the transition should occur at $A \approx L^{4}$. To see this, we first observe (2.3) and a standard argument (see for instance $[7,19]$ ) will quickly show that the time $X$ takes to exit the big domain $\Omega$ is comparable to the time the level set averaged diffusion takes takes to exit one cell. Consequently, $\bar{\tau} \approx O(1)$ on cell interiors. Now one would expect that the transition between the homogenized and averaged regimes occurs when the homogenized expected exit time $L^{2} / D_{\text {eff }}$ is comparable to the averaged expected exit time $O(1)$. Since $D_{\text {eff }} \approx \sqrt{A}$, we expect the transition to occur when $L^{2} \approx \sqrt{A}$, as seen in Theorem 2.1.

\section{Numerical Results in the CRitical Regime.}

In this section we present our results for the exit time in the critical regime. We begin with Figure 2, a plot of $\bar{\tau}(L / 2, L / 2)$ vs $L$ for values of $L$ ranging from 10 to 80 , with $A=L^{4}$ and the domain $\Omega$ to be a square of side length $L$ (left) or a circle of diameter $L$ (right). The graph was computed using a Monte-Carlo simulation using 10,000 realizations. The numerical method used is based on [16,17], and is described in Section 5 .

To see that the mean exit time agrees with that of the homogenized process, we recall that the asymptotic behaviour of the effective diffusion matrix is

$$
D_{\text {eff }}=D_{\text {eff }}(A) \stackrel{\text { def }}{=} \lim _{t \rightarrow \infty} \frac{E X_{t} \otimes X_{t}}{t} \approx c_{0} \sqrt{A} I
$$

and we numerically compute $c_{0} \approx 0.6056$. Now, the expected exit time of the homogenized process $\sqrt{D_{\text {eff }}} W$ from the center of a circle of diameter $L=A^{1 / 4}$ can be (analytically) computed to be $1 /\left(8 c_{0}\right) \approx 0.2064$, which agrees well with Figure 2(b). For the square of side length $L$, a numerical simulation shows the 


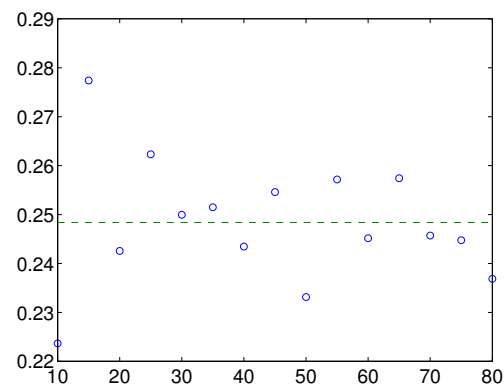

(a) Square of side length $L$

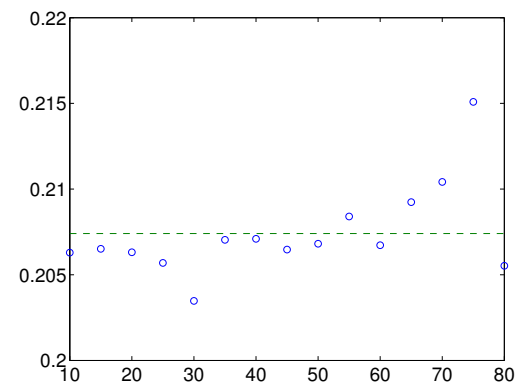

(b) Circle of diameter $L$

Figure 2. Expected exit time of $X$ for different values of $L$, and $A=L^{4}$.

expected exit time of $\sqrt{D_{\text {eff }}} W$ from the center is $0.1473 / c_{0} \approx 0.2433$, which agrees well with Figure 2(a).

We now fix $L=40, A=L^{4}$ and consider the exit time $\tau$ of the process $X$ from the center of a square of side length $L$, and a circle of diameter $L$. We know that with small probability, some trajectories of $X$ will travel ballistically along separatrices and exit the domain very quickly. To account for this, we ignore all trajectories of $X$ that exit the domain in time less than a small time threshold (roughly 0.03 seconds). For the remaining trajectories, we compare the distribution $\tau$ to $\tau_{\text {eff }}$, the exit time of the effective Brownian motion $\sqrt{D_{\text {eff }} W}$. Our results show the distributions are almost identical, and are illustrated in Figure 3. Specifically, Figure 3(a) shows the cumulative distribution functions of $\tau$ and $\tau_{\text {eff }}$ from a square of side length 40, and Figure 3(b) shows the cumulative distribution functions of $\tau$ and $\tau_{\text {eff from a circle of diameter } 40 .}$

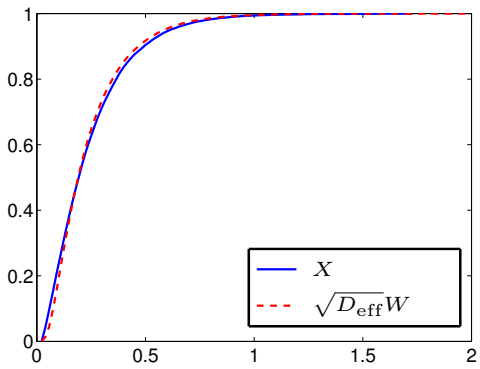

(a) Square of side length 40

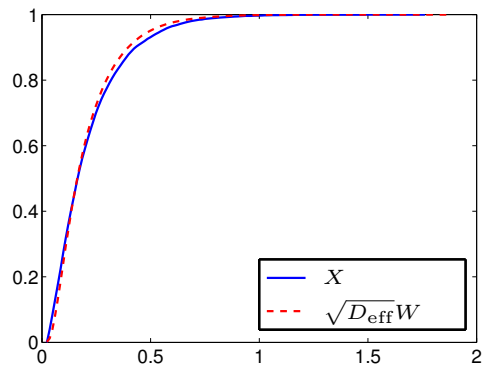

(b) Circle of diameter 40

Figure 3. CDF for $\tau$ compared with the CDF of the exit time of the effective Brownian motion. 


\section{NumERICAL RESUlTS IN THE HOMOGENIZATION REGIME}

A fundamental question left unresolved by [9] is about the asymptotic profile of $\bar{\tau}$ in any domain which is not a disk. This roughly translates to the question of whether 'homogenization works' when the asymptotic profile is not quadratic. We explain the theoretical obstruction, and our numerical findings in this section.

The proof of Theorem 2.1 in the homogenization regime (i.e. $A \ll L^{4}$ ) uses an asymptotic expansion. To carry this out, we rescale the domain to a square of side length 1 which we denote by $\Omega_{1}$. Let $\varepsilon=1 / L$, and $\bar{\sigma}=\bar{\sigma}_{A, \varepsilon}$ be the rescaled function defined by $\bar{\sigma}(x)=\varepsilon^{2} \bar{\tau}(x / \varepsilon)$. Then, $\bar{\sigma}$ satisfies the equation

$$
\left\{\begin{aligned}
-\frac{1}{2} \Delta \bar{\sigma}+\frac{A}{\varepsilon} v\left(\frac{x}{\varepsilon}\right) \cdot \nabla \bar{\sigma} & =1 & & \text { in } \Omega_{1} \stackrel{\text { def }}{=}(0,1)^{2}, \\
\bar{\sigma} & =0 & & \text { on } \partial \Omega_{1}
\end{aligned}\right.
$$

Now consider a multi-scale expansion of an approximate solution $\tilde{\sigma}$ up to two terms

$$
\tilde{\sigma}(x)=\sigma_{0}(x)+\varepsilon \sigma_{1}(x, y)+\varepsilon^{2} \sigma_{2}(x, y), \quad \text { where } y=\frac{x}{\varepsilon} \text { is the 'fast' variable. }
$$

The usual practice in homogenization $[1,15]$ is to choose $\sigma_{0}$ to be a solution of the effective problem, and then choose $\sigma_{1}, \sigma_{2}$ to be periodic, mean zero in the fast variable and satisfy equations that balance the $O(\varepsilon)$ and $O(1)$ terms respectively.

While this works perfectly well for $A$ fixed, the proofs in [9] will only work in an exceptional situation if $A \rightarrow \infty$. To elaborate on this, choosing $\sigma_{1}, \sigma_{2}$ as described above, we will obtain

$$
\begin{aligned}
& -\frac{1}{2} \Delta \tilde{\sigma}+\frac{A}{\varepsilon} v\left(\frac{x}{\varepsilon}\right) \cdot \nabla \tilde{\sigma}=-\Delta\left(\sigma_{0}+\varepsilon \sigma_{1}+\varepsilon^{2} \sigma_{2}\right)+ \\
& A\left\langle v \cdot \nabla_{x} \sigma_{1}\right\rangle-2 \varepsilon \nabla_{x} \cdot \nabla_{y} \sigma_{2}+\varepsilon A v \cdot \nabla_{x} \sigma_{2},
\end{aligned}
$$

where $\langle\cdot\rangle$ denotes the average with respect to the fast variable. When $A$ is fixed, all terms with an $\varepsilon$ are harmless, and the term $A\left\langle v \cdot \nabla_{x} \sigma_{1}\right\rangle$ can be computed explicitly [5,13]. When we additionally have $A \rightarrow \infty$, the presence of the term $\varepsilon A v$. $\nabla_{x} \sigma_{2}$ is catastrophic! Fortunately this term identically vanishes, in the exceptional situation that $\sigma_{0}$ is quadratic. It is this exceptional situation that [9] heavily exploits in the proof.

Observe that if one replaces $\Omega_{1}$ with $B(0,1)$ in $(4.1)$, then $\sigma_{0}$ must be quadratic (explicitly, $\sigma_{0}=\frac{1}{2 \operatorname{tr}\left(D_{\text {eff }}(A)\right)}\left(1-|x|^{2}\right)$, where $D_{\text {eff }}(A)$ is the effective diffusivity matrix). In this case, the result in $(2.2)$ can be considerably improved. For convenience, we state the improved result in rescaled coordinates.

Theorem 4.1 ([9]). Suppose $\bar{\sigma}^{\prime}$ is the solution to (4.1) on $B(0,1)$, the disk of radius 1 . Suppose for some $\alpha>0, A=O\left(1 / \varepsilon^{4-\alpha}\right)$ as $\varepsilon \rightarrow 0$. Then there exists $c=c(\alpha)>0$, independent of $A, \varepsilon$ such that for all $\varepsilon$ sufficiently small,

$$
\left\|\bar{\sigma}-\sigma_{0}\right\|_{L^{\infty}} \leqslant c \frac{\varepsilon}{A^{1 / 4}}
$$

where $\sigma_{0}=\sigma_{0}(A)$ is the solution of the effective problem

$$
-\nabla \cdot D_{\text {eff }}(A) \nabla \sigma_{0}=1 \quad \text { in } B(0,1) \quad \text { and } \quad \sigma_{0}=0 \quad \text { on } \partial B(0,1) \text {. }
$$

Observe that $D_{\text {eff }}(A)=O(\sqrt{A})$, and hence $\sigma_{0}=O(1 / \sqrt{A}) \rightarrow 0$; however the right hand side of $(4.2)$ is $\varepsilon / A^{1 / 4}=o(1 / \sqrt{A})$ by assumption. This means that the limiting profile of $\sqrt{A} \bar{\sigma}$ is exactly $\sqrt{A} \sigma_{0}$, which is finite and non-zero in the interior 
of $B(0,1)$. On the other hand, equation $(2.2)$ provides only upper and lower bounds for the limiting profile of $\bar{\sigma}$.

We numerically confirm that $(4.2)$ is valid in a situation where $\sigma_{0}$ is not quadratic. For this, we return to studying the (rescaled) expected exit time $\bar{\sigma}$ on $\Omega_{1}$, the square of side length 1 . Figure 4(a) shows a slices of the graphs of $\bar{\sigma}$ and $\sigma_{0}$ along the diagonal of the square $\Omega_{1}$. Figure 4(b) shows slices of the graphs of $\bar{\sigma}$ and $\sigma_{0}$ along a horizontal line through the center. Figures $4(\mathrm{c})$ and $4(\mathrm{~d})$ show the same slices on a $1.5 \times 2.5$ rectangle, instead of the square $\Omega_{1}$. We observe a remarkable agreement between $\bar{\sigma}$ and $\bar{\tau}_{0}$, suggesting that $(4.2)$ is valid for general $\sigma_{0}$, despite apparent theoretical obstacles arising from the catastrophic $\varepsilon A v \cdot \nabla_{x} \sigma_{2}$ term.

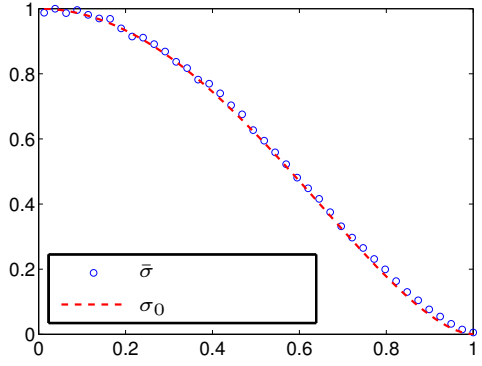

(a) Sliced along the diagonal

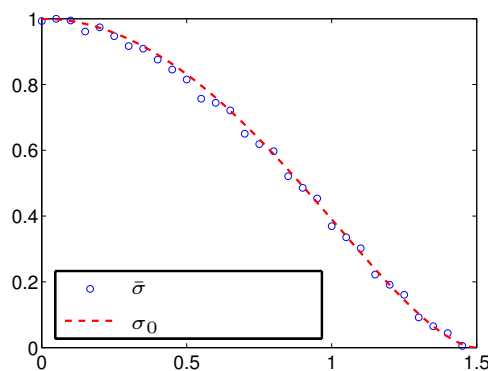

(c) Sliced along the diagonal

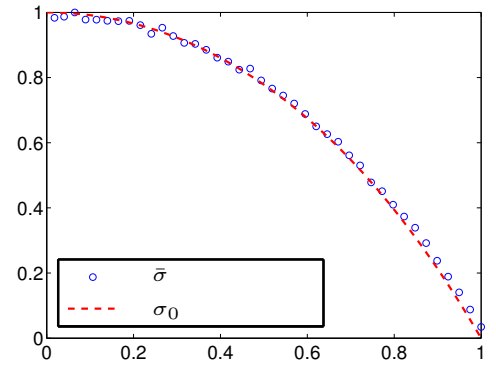

(b) Sliced horizontally through the center.

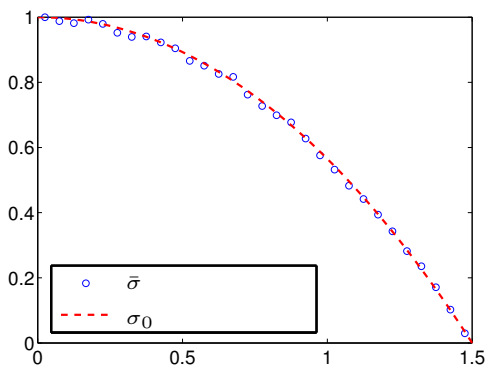

(d) Sliced horizontally through the center.

Figure 4. Slices of the graphs of $\bar{\sigma}$ and $\sigma_{0}$ in a square (top) and rectangle (bottom).

\section{Numerical Method}

We conclude this paper with a description of the numerical method used for our simulations. As mentioned earlier, the Euler-Maruyama is not suitable for our purposes, and the method we use is that in [16]. We describe the method here, and present a simplified analysis of it based on the theory of modified equations. 
We first rescale time by a factor of $1 / A$, and consider the process $Y_{t} \stackrel{\text { def }}{=} X_{t / A}$. With this change, SDE (1.1) becomes

$$
d Y_{t}=v\left(Y_{t}\right) d t+\frac{1}{\sqrt{A}} d W_{t}^{\prime}
$$

where $W_{t}^{\prime}=\sqrt{A} W_{t / A}$ is a new Brownian motion.

Observe that for large $A$, the $\mathrm{SDE}(5.1)$ is a small random perturbation of the Hamiltonian system

$$
\frac{d Y}{d t}=v(Y)
$$

with Hamiltonian $H$ (see equation $(1.2)$ ). So it is natural to look for numerical schemes which respect features of the underlying deterministic dynamics [8]. The scheme we use is based on the (deterministic) scheme in [17].

Define $d_{i}, e_{i}$ by

$$
d_{1}=\left(\begin{array}{r}
-1 / 2 \\
1 / 2
\end{array}\right), \quad d_{2}=\left(\begin{array}{l}
-1 / 2 \\
-1 / 2
\end{array}\right), \quad e_{1}=2 \pi\left(\begin{array}{l}
1 \\
1
\end{array}\right), \quad e_{2}=2 \pi\left(\begin{array}{c}
1 \\
-1
\end{array}\right),
$$

and observe

$$
v=\sum_{i=1}^{2} v_{i}, \quad \text { where } v_{i}(x)=d_{i} g\left(\left\langle e_{i}, x\right\rangle\right), \text { and } g(x)=\sin (x) .
$$

Here $\langle\cdot, \cdot\rangle$ denotes the standard inner product on $\mathbb{R}^{2}$.

The main idea behind the scheme in [17] is that each constituent vector field $v_{i}$ is divergence free, and can be explicitly integrated. Namely, in view of the identities,

$$
\left\langle d_{i}, e_{i}\right\rangle=\left\langle d_{i}, d_{j}\right\rangle=\left\langle e_{i}, e_{j}\right\rangle=0, \quad \text { for } i, j \in\{1,2\}, \text { with } i \neq j,
$$

we see

$$
\begin{aligned}
\dot{Z}_{t}(z) & =v_{i}\left(Z_{t}(z)\right) \quad \Longleftrightarrow \quad Z_{t}(z)=z+t d_{i} g\left(\left\langle e_{i}, z\right\rangle\right) . \\
\& \quad Z_{0}(z) & =z
\end{aligned}
$$

In view of (5.4), using the Euler method to integrate each constituent field $v_{i}$ will give an explicit, volume preserving, numerical integrator for $v$. Of course, volume preserving integrators cannot also preserve the Hamiltonian unless they coincide with the exact flow [3]. However, this numerical method preserves a modified Hamiltonian (discussed later), preventing it from "spiraling outward", which is usually the difficulty encountered when the Euler method is used to integrate (5.2) over long time intervals.

In the stochastic setting, we choose a sequence of independent random variables $\left(\xi_{n}\right)$ so that each $\xi_{n}$ is a two dimensional normally distributed random variable with mean 0 and covariance matrix $I$. Let $\Delta t>0$ be the time step, and $y \in \mathbb{R}^{2}$ be the initial condition. We define $Y^{n}$, an approximation to the solution of (5.1) at time $n \Delta t$, by

$$
\left\{\begin{array}{c}
Z_{1}^{n}=Y^{n}+\Delta t d_{1} g\left(\left\langle Y^{n}, e_{1}\right\rangle\right), \quad Z_{2}^{n}=Z_{1}^{n}+\Delta t d_{2} g\left(\left\langle Z_{1}^{n}, e_{2}\right\rangle\right) \\
Y^{n+1}=Z_{2}^{n}+\left(\frac{\Delta t}{A}\right)^{1 / 2} \xi_{n},
\end{array}\right.
$$

with $Y^{0}=y$. It quickly follows from $(5.4)$ that the map $y \mapsto Y^{n}$ is (surely) volume preserving for all $n$. Thus (5.5) gives us a volume preserving numerical scheme for (5.1), whose inviscid counterpart does not spiral outward! 
5.1. A analysis of the method using modified equations. A simple analysis of the qualitative features of our numerical scheme can be obtained using the theory of modified equations for SDEs [20,23]. In particular, assume that equation (5.1) is solved by a first order weak method, as for example the stochastic splitting method (5.5). Then one looks for coefficients $u_{1}(x), \sigma_{1}(x)$, such that (5.5) approximates

$$
d Y_{t}=\left[u\left(Y_{t}\right)+\Delta t u_{1}\left(Y_{t}\right)\right] d t+\left[\frac{1}{\sqrt{A}}+\Delta t \sigma_{1}\left(Y_{t}\right)\right] d W_{t}
$$

to second weak order. The coefficients $u_{1}, \sigma_{1}$ can calculated following [20], when the numerical method used is the Euler-Maruyama method. For more complicated numerical integrators a more general framework is developed in [23].

The underlying idea for calculating these coefficients is that $u_{1}, \sigma_{1}$ should be chosen such that the 'modified' local error between the numerical method and the solution of (5.6) should be one order higher than the 'original' local error between the numerical method and the solution of (5.1). This is primarily accomplished using weak stochastic Taylor expansions [18]. In our situation, $u_{1}$ and $\sigma_{1}$ can be calculated using equation (3.9) in [23], which shows that (5.5) approximates

$$
d Y_{t}=\nabla^{\perp} \tilde{H}\left(Y_{t}\right) d t+\frac{1}{\sqrt{A}}\left(I-\frac{\Delta t}{2} \nabla v\left(Y_{t}\right)\right) d W_{t}
$$

weakly to second order. Here $v$ is our velocity field from $(\overline{1.2)}$, and $\tilde{H}$ is the modified Hamiltonian defined by

$$
\tilde{H}=H(x)\left(1+\frac{2 \pi^{2} \Delta t}{A}\right)+\Delta t H_{1}(x),
$$

where

$$
H_{1}\left(x_{1}, x_{2}\right)=\frac{1}{16 \pi^{2}}\left(\cos 4 \pi x_{2}-\cos 4 \pi x_{1}\right) .
$$

Observe that the modified equation (5.7) is a random perturbation of a two dimensional Hamiltonian flow with a cellular Hamiltonian $\tilde{H}$. It is easy to see that as long as $\Delta t=o(1)$, solutions to (5.7) approximate solutions to (5.1). Consequently, this means our method will integrates $(1.1)$ on the time interval $[0,1]$ in roughly $A$ steps. The Euler-Maruyama method, in contrast, will require roughly $A^{2}$ steps. Finally, we remark that when comparing the modified equation (5.7) with the one that one gets from the deterministic analysis [8], the only extra term that appears in $\tilde{H}$ is $\frac{2 \pi^{2} \Delta t}{A} H$, which accounts for the presence of noise in the problem.

\section{REFERENCES}

[1] A. Bensoussan, J.-L. Lions, and G. Papanicolaou, Asymptotic analysis for periodic structures, Studies in Mathematics and its Applications, vol. 5, North-Holland Publishing Co., Amsterdam, 1978. MR503330 (82h:35001)

[2] H. Berestycki, F. Hamel, and N. Nadirashvili, Elliptic eigenvalue problems with large drift and applications to nonlinear propagation phenomena, Comm. Math. Phys. 253 (2005), no. 2, 451-480. MR2140256 (2006b:35057)

[3] P. Chartier, E. Faou, and A. Murua, An algebraic approach to invariant preserving integrators: the case of quadratic and Hamiltonian invariants, Numer. Math. 103 (2006), no. 4, 575-590, DOI 10.1007/s00211-006-0003-8. MR2221062 (2007b:37209)

[4] S. Childress, Alpha-effect in flux ropes and sheets, Phys. Earth Planet Inter. 20 (1979), 172180. 
[5] A. Fannjiang and G. Papanicolaou, Convection enhanced diffusion for periodic flows, SIAM J. Appl. Math. 54 (1994), no. 2, 333-408, DOI 10.1137/S0036139992236785. MR1265233 (95d:76109)

[6] A. Fannjiang, A. Kiselev, and L. Ryzhik, Quenching of reaction by cellular flows, Geom. Funct. Anal. 16 (2006), no. 1, 40-69, DOI 10.1007/s00039-006-0554-y. MR2221252 (2007e:35157)

[7] M. I. Freidlin and A. D. Wentzell, Random perturbations of dynamical systems, 2nd ed., Grundlehren der Mathematischen Wissenschaften [Fundamental Principles of Mathematical Sciences], vol. 260, Springer-Verlag, New York, 1998. Translated from the 1979 Russian original by Joseph Szücs. MR1652127 (99h:60128)

[8] E. Hairer, C. Lubich, and G. Wanner, Geometric numerical integration, 2nd ed., Springer Series in Computational Mathematics, vol. 31, Springer-Verlag, Berlin, 2006. Structurepreserving algorithms for ordinary differential equations.

[9] G. Iyer, T. Komorowski, A. Novikov, and L. Ryzhik, From Homogenization to Averaging in Cellular Flows, preprint, available at arXiv:1108.0074.

[10] V. V. Jikov, S. M. Kozlov, and O. A. Oleŭnik, Homogenization of differential operators and integral functionals, Springer-Verlag, Berlin, 1994. Translated from the Russian by G. A. Yosifian [G. A. Iosif'yan]. MR1329546 (96h:35003b)

[11] A. Kiselev and L. Ryzhik, Enhancement of the traveling front speeds in reaction-diffusion equations with advection, Ann. Inst. H. Poincaré Anal. Non Linéaire 18 (2001), no. 3, 309358, DOI 10.1016/S0294-1449(01)00068-3 (English, with English and French summaries). MR1831659 (2002c:35155)

[12] L. Koralov, Random perturbations of 2-dimensional Hamiltonian flows, Probab. Theory Related Fields 129 (2004), no. 1, 37-62, DOI 10.1007/s00440-003-0320-0. MR2052862 (2005e:60179)

[13] A. Novikov, G. Papanicolaou, and L. Ryzhik, Boundary layers for cellular flows at high Péclet numbers, Comm. Pure Appl. Math. 58 (2005), no. 7, 867-922, DOI 10.1002/cpa.20058. MR2142878 (2007m:76045)

[14] B. Øksendal, Stochastic differential equations, 6th ed., Universitext, Springer-Verlag, Berlin, 2003. An introduction with applications. MR2001996 (2004e:60102)

[15] G. A. Pavliotis and A. M. Stuart, Multiscale methods, Texts in Applied Mathematics, vol. 53, Springer, New York, 2008. Averaging and homogenization. MR2382139 (2010a:60003)

[16] G. A. Pavliotis, A. M. Stuart, and K. C. Zygalakis, Calculating effective diffusiveness in the limit of vanishing molecular diffusion, J. Comput. Phys. 228 (2009), no. 4, 1030-1055, DOI 10.1016/j.jcp.2008.10.014. MR2489942 (2010d:76071)

[17] G. R. W. Quispel and D. I. McLaren, Explicit volume-preserving and symplectic integrators for trigonometric polynomial flows, J. Comput. Phys. 186 (2003), no. 1, 308-316, DOI 10.1016/S0021-9991(03)00068-8. MR1967370 (2004c:37204)

[18] A. Rößler, Stochastic Taylor expansions for the expectation of functionals of diffusion processes, Stochastic Anal. Appl. 22 (2004), no. 6, 1553-1576, DOI 10.1081/SAP-200029495. MR2095070 (2005m:60151)

[19] P. B. Rhines and W. R. Young, How rapidly is passive scalar mixed within closed streamlines?, J. Fluid Mech. 133 (1983), 135-145.

[20] T. Shardlow, Modified equations for stochastic differential equations, BIT 46 (2006), no. 1, 111-125, DOI 10.1007/s10543-005-0041-0. MR2214851 (2006k:65017)

[21] W. Young, A. Pumir, and Y. Pomeau, Anomalous diffusion of tracer in convection rolls, Phys. Fluids A 1 (1989), no. 3, 462-469, DOI 10.1063/1.857415. MR1021635 (90i:76133)

[22] A. Zlatoš, Reaction diffusion front speed enhancement by flows, Ann. Inst. H. Poincar Anal. Non Linaire 28 (2011), 711-726, to appear.

[23] K. C. Zygalakis, On the existence and the applications of modified equations for stochastic differential equations, SIAM J. Sci. Comput. 33 (2011), no. 1, 102-130, DOI 10.1137/090762336. MR2783188 
NUMERICAL STUDIES OF HOMOGENIZATION UNDER A FAST CELLULAR FLOW. 11

Department of Mathematical Sciences, Carnegie Mellon University, Pittsburgh PA 15213

E-mail address: gautam@math.cmu.edu

Mathematical Institute, University of Oxford, 24-29 St Giles', OX13LB

E-mail address: zygalakis@maths.ox.ac.uk 